\title{
Photoresponse of Long-Wavelength AlGaAs/GaAs Quantum Cascade Detectors
}

\author{
Liang $\mathrm{Li}^{1}$ and Dayuan Xiong ${ }^{1,2}$ \\ ${ }^{1}$ Key Laboratory of Polar Materials and Devices, Ministry of Education, East China Normal University, Shanghai 200241, China \\ ${ }^{2}$ Shanghai Key Laboratory of Multidimensional Information Processing, East China Normal University, Shanghai 200241, China
}

Correspondence should be addressed to Dayuan Xiong; dyxiong@ee.ecnu.edu.cn

Received 27 November 2014; Accepted 4 January 2015

Academic Editor: Wen Lei

Copyright (C) 2015 L. Li and D. Xiong. This is an open access article distributed under the Creative Commons Attribution License, which permits unrestricted use, distribution, and reproduction in any medium, provided the original work is properly cited.

\begin{abstract}
We study the photoresponse and photocurrents of long-wavelength infrared quantum cascade detectors (QCDs) based on $\mathrm{AlGaAs} / \mathrm{GaAs}$ material system. The photocurrent spectra were measured at different temperatures from $20 \mathrm{~K}$ to $100 \mathrm{~K}$ with a low noise Fourier transforming infrared spectrometer. The main response peak appeared at $8.9 \mu \mathrm{m}$ while four additional response peaks from $4.5 \mu \mathrm{m}$ to $10.1 \mu \mathrm{m}$ were observed as well. We confirmed that the photocurrent comes from phonon assisted tunneling and the multipeak behavior comes from the complicated optical transition in the quantum cascade structure. This work is valuable for the future design and optimization of QCD devices.
\end{abstract}

\section{Introduction}

Quantum cascade detectors (QCDs) are photovoltaic detectors. They can operate under zero bias applied and consequently do not suffer any dark current which is expected as a promising alternative of quantum well infrared photodetectors (QWIPs) [1, 2]. For focal plane array (FPA) application, the QCDs perform pure photoresponse current to reach a longer integration time [3] and lower noise equivalent temperature difference (NETD) [4]. Based on bound-tobound intraband transition, the line width of QCDs is narrow, which means high selectivity to wavelength. Moreover, QCDs have been shown to work at higher temperature [5] and been reported in different materials and all infrared atmospheric windows [5-9].

To prevent false-alarm and improve detection accuracy, two color FPAs were studied and fabricated [10-12]. Conventionally, two-color detection is achieved by two single-color detectors fabricated together. So, secondary photoresponse peaks are undesired which would act as cross talk and noise in response spectra. QCDs perform excellent for the narrowband detection. But confined with their multisized quantum wells, the photoexcited transitions are complicated. As a result, several secondary peaks appear. To eliminate cross talk and further improve the detector's performance, detailed study on the secondary peaks is of particularly importance.

\section{Materials and Methods}

The samples under study are GaAs/AlGaAs QCDs designed to operate at $8.9 \mu \mathrm{m}[3,13]$. The wafer used to fabricate this QCD sample was grown by molecular beam epitaxy $(\mathrm{MBE})$, and the layer sequence from substrate to top was $400 \mathrm{~nm}$ n-doped GaAs bottom contact (Si-doped to $1 \times$ $10^{18} \mathrm{~cm}^{-3}$ ), 40 periods of 7 coupled $\mathrm{Al}_{0.33} \mathrm{Ga}_{0.67} \mathrm{As} / \mathrm{GaAs}$ superlattice active region, and $350 \mathrm{~nm}$ n-doped GaAs top contact in which doping concentration is equal to the bottom contact. The first QW of each period absorbs incident light and is $\mathrm{n}$-doped with the doping concentration $5 \times$ $10^{17} \mathrm{~cm}^{-3}$. The thickness of different QWs and barriers is $68 / 56.5 / 20 / 39.55 / 23 / 31 / 28 / 31 / 34 / 31 / 38 / 31 / 48 / 22.6(\AA)$ in roman and bold, respectively. The mesa size is $200 \times 200 \mu \mathrm{m}^{2}$ and electrical contacts were formed by a standard lift-off deposition of $\mathrm{AuGe} / \mathrm{Ni} / \mathrm{Au}(100 / 20 / 400 \mathrm{~nm})$. To theoretically describe the electronic properties of QCDs, we applied the standard eight-band $k \cdot p$ model. As shown in Figure 1, the electrons which the energy $h v=E_{8}-E_{1}$ could be excited from $E_{1}$ to $E_{8}$. $E_{7}$ is designed to equal $E_{8}$ which 


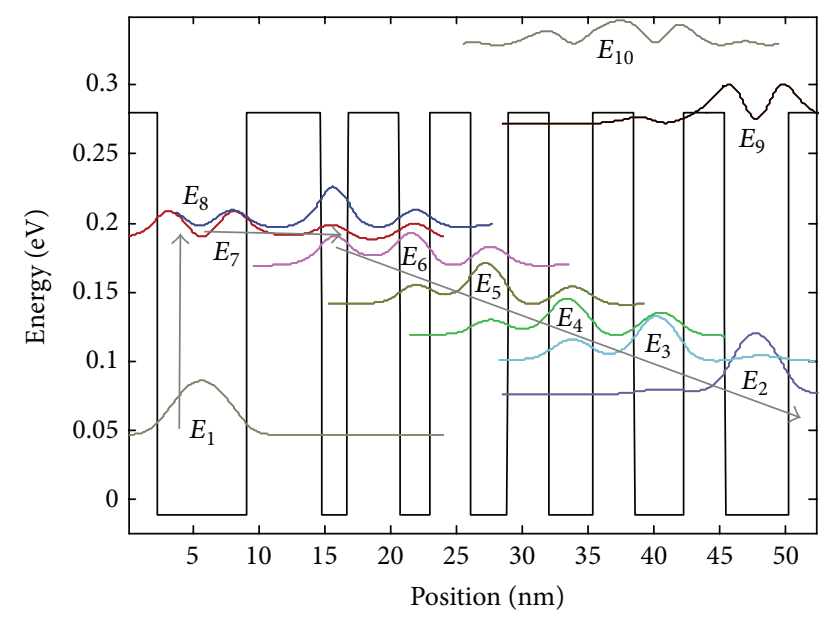

FIGURE 1: Self-consisted calculated conduction band structure of one period.

could allow the exited electrons resonant tunneling to reduce the recombination possibility and contribute to effective transport. Then, excited electrons tended to transport in the direction $E_{7} \rightarrow E_{6} \rightarrow E_{5} \rightarrow E_{4} \rightarrow E_{3} \rightarrow E_{2} \rightarrow E_{1, \text { next-period }}$ by LO-phonon assisted tunneling due to the coupling of wavefunction of adjacent wells.

\section{Results and Discussion}

Considering the complicated interactions between the intersubbands in QCDs, the absorption and response spectrum is not so pure and sensitive to $8.9 \mu \mathrm{m}$ only. With the diffusion and tunneling of electrons, more than one absorption process could be figured out theoretically as Figure 2(a) dashed line indicated. Besides, we measured response photocurrent spectra under zero bias voltage at different temperature. QCD sample is soldered on a copper heat sink and placed in a closed cycle helium cryostat. The measurements were performed by using a SR570 low noise current preamplifier and a Fourier transform infrared spectrometer (Nicolet 6700). Response photocurrent spectra at different temperatures are shown as solid lines in Figure 2(a).

Three clear photoresponse peaks were found immediately in Figure 2(a) which are $E_{1} \rightarrow E_{7} / E_{8}, E_{2} \rightarrow E_{8}$, and $E_{1} \rightarrow$ $E_{9}$ processes. Other two peaks could be seen after zooming in which are corresponding to $E_{2} \rightarrow E_{9}$ and $E_{2} \rightarrow E_{10}$ transitions. It is to be noticed that the valley at $1250 \mathrm{~cm}^{-1}$ is a background absorption so that the peak at $1270 \mathrm{~cm}^{-1}$ is actually a fake one.

To further improve the detector's performance, detailed study on the formation of multipeak spectra is needed. As is known, photocurrent $I_{p}$ could be expressed as [14]

$$
I_{p}=n_{p} e v,
$$

where $n_{p}$ is the quantity of photogenerated carriers, $e$ is electron charge, and $v$ is the transport velocity. Moreover, for a certain wavelength $\lambda$, the photocurrent could be given by

$$
I_{p}(\lambda)=n_{p}(\lambda) \operatorname{ev}(\lambda) .
$$

Equations (1) and (2) are true when the electron concentration is high enough to be photoexcited. Otherwise, the $I_{p}$ is also restricted to electron concentration. Besides, corresponding to different photoexcited transitions, the transport paths are not all the same in QCDs, which would lead to different $v(\lambda)$. At low temperature condition, the impurities are ionized less and mainly distributed in $E_{1}$ subband. Thus the optical excited transitions primarily occur from $E_{1}$ to $E_{7}, E_{8}$, and $E_{9}$, respectively. With temperature rises, more impurities will be ionized and electron concentration will get higher. Consequently, more quantum states of $E_{1}$ subband would be occupied. According to Pauli exclusion principle and Hund's rules, electrons would tend to be distributed at higher energy levels such as $E_{2}$ by means of diffusion and tunneling. Then, optical transition from $E_{2}$ is enhanced as Figures 2(b) and 2(c) indicated.

The calculated electrons distribution at different temperatures is presented in Figure 3. It can be seen that electron density at $E_{2}$ subband is hard to be identified at $50 \mathrm{~K}$, while the density at $E_{2}$ or even other subbands becomes larger with temperature increases. At temperature higher than $40 \mathrm{~K}$, the electron density grows exponentially rapidly as the inset Figure 3 indicated, and corresponding to Figure 2(b), the response of $E_{2} \rightarrow E_{8}$ transition becomes clear at $40 \mathrm{~K}$. This indicated that our QCD can be operated around $80 \mathrm{~K}$, but the electron concentration of $E_{2}$ grows to ten times as that at $40 \mathrm{~K}$ which would be a nonignorable cross talk.

In addition, band-gap and barrier height would be influenced by temperature. Energy levels would be rearranged by structure variation of quantum wells, especially the quasibound energy levels such as $E_{9}$. As for photoconductive QWIPs working at nonzero applied bias, the bound-to-quasibound transition appears redshift with temperature rises [15]. But for QCDs, null bias working condition makes the transport mechanisms different. As is expected, $E_{1} \rightarrow E_{9}$ transition appears blueshift with temperature getting higher as Figure 2(d) indicated. The experiment results and calculated energy gap between $E_{1}$ and $E_{9}$ are shown in Figure 4. A general agreement has been obtained.

From responsivity and dark current, we can deduce the Johnson noise limited detectivity given by [2]

$$
D^{*}=R(\lambda) \sqrt{\frac{R_{0} A}{4 k T}},
$$

where $R(\lambda)$ is the peak responsivity, $R_{0}$ is the device resistance at null bias, $A$ is the mesa surface, and $T$ is the temperature of the sample. We obtained $D^{*}(\lambda=8.9)=1.4 \times 10^{10}$ Jones at $80 \mathrm{~K}$ as Figure 5 presented. Moreover, after the elimination of secondary response peaks, the $g$ factor in spectra would get higher, leading to a higher $D^{*}$.

In our QCD detector samples, the $E_{1} \rightarrow E_{7}$ and $E_{1} \rightarrow E_{8}$ transitions are expected to be the primary optical transitions. The spectra noises which act as cross talk could be classified in two categories. The first category is the transitions from $E_{1}$ to non- $E_{7} / E_{8}$ subbands such as $E_{1} \rightarrow E_{9}$ and the second is excited from non- $E_{1}$ (for example, $E_{2} \rightarrow E_{8}$ ) energy levels. For the first category, thicker barriers would reduce the overlap of wavefunctions, leading to a lower transition 


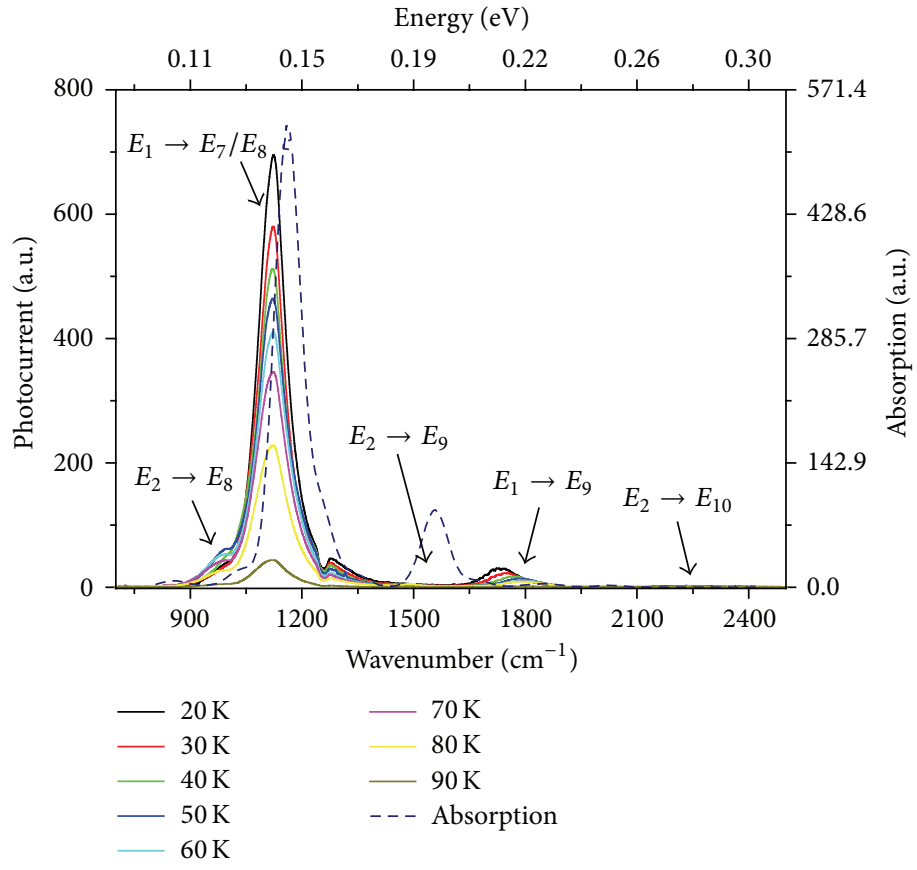

(a)
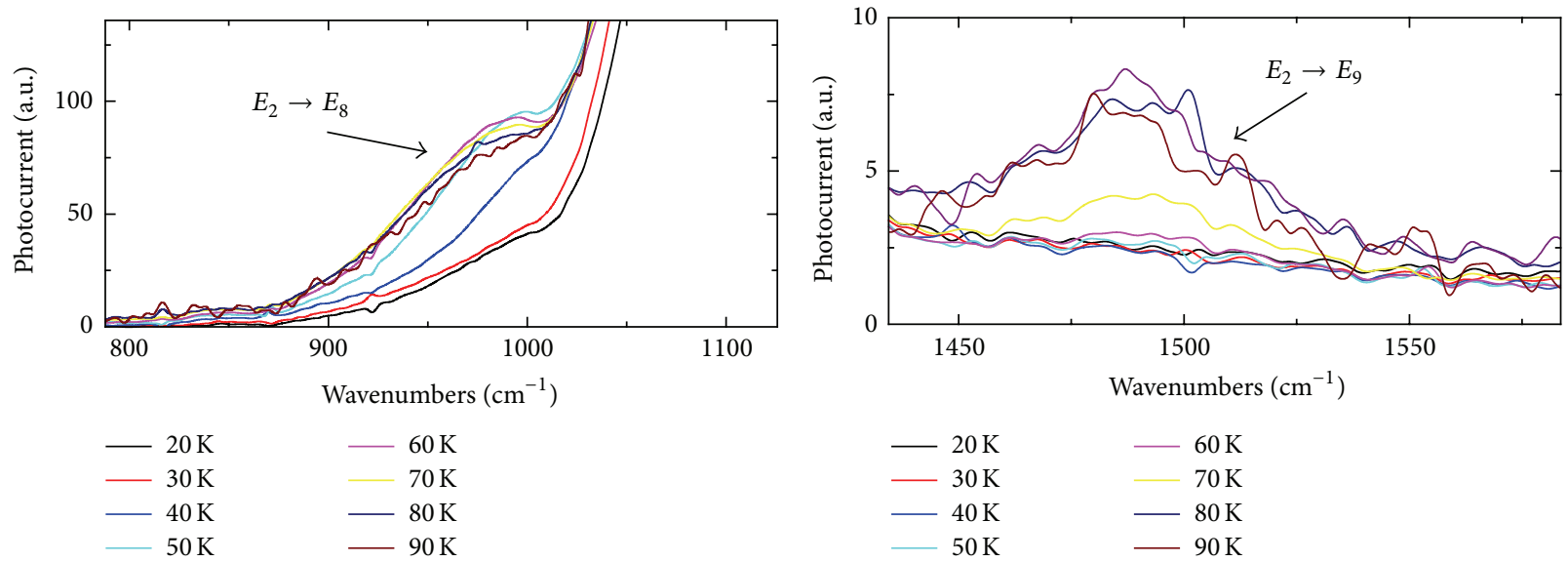

(b)

(c)

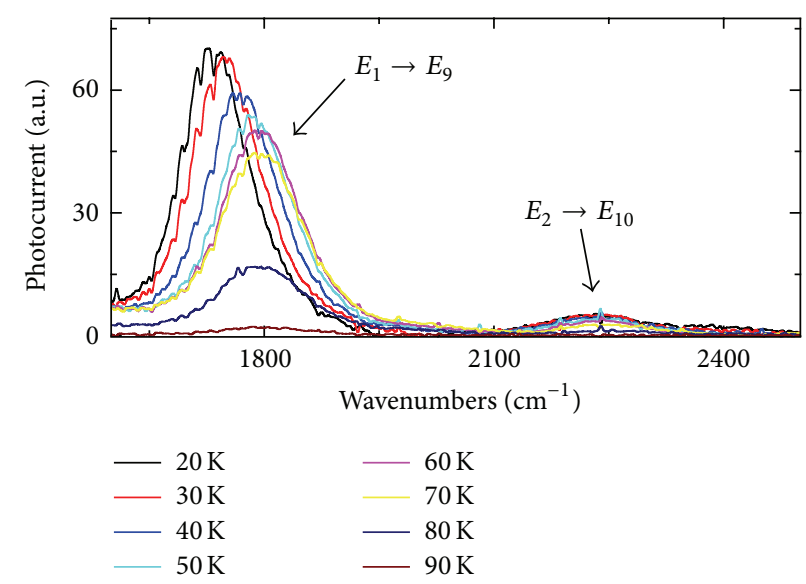

(d)

Figure 2: (a) Calculated absorption spectra at $300 \mathrm{~K}$ (dashed line) and measured photoresponse spectra (solid line). (b) Details (normalized) around $E_{2} \rightarrow E_{8}$. (c) Details (normalized) around $E_{2} \rightarrow E_{9}$. (d) Details around $E_{1} \rightarrow E_{9}$ and $E_{2} \rightarrow E_{10}$. 


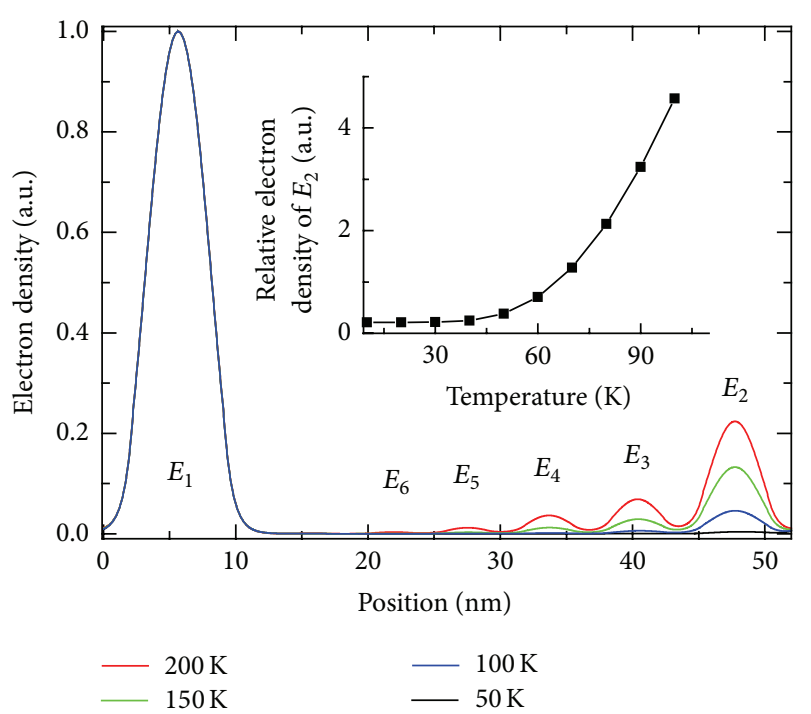

Figure 3: Calculated electron density at different temperatures (normalized). Inset is the electron concentration ratio of $E_{2}$ and $E_{1}$ subband.

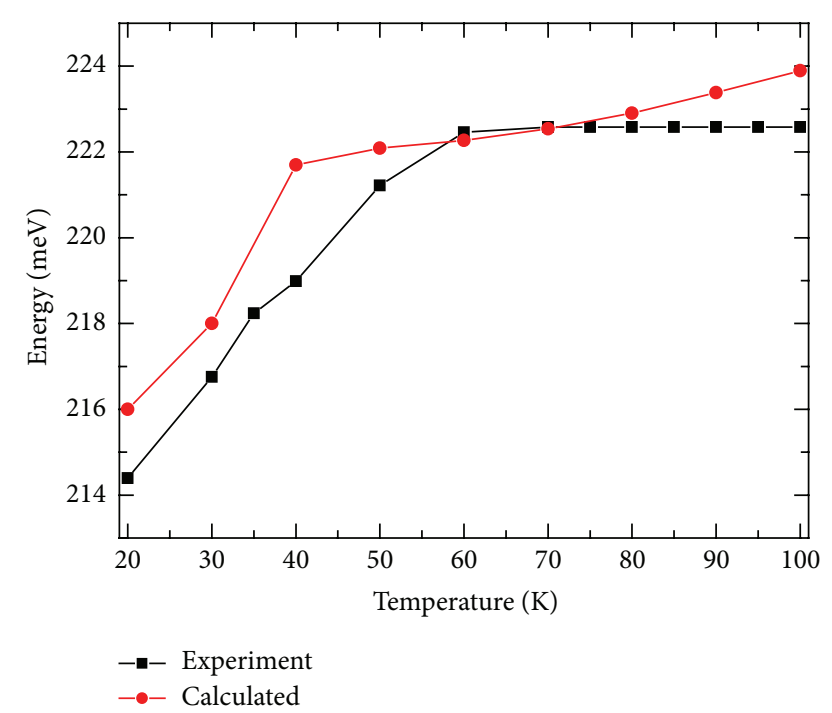

Figure 4: Calculated and experiment $E_{1} \rightarrow E_{9}$ transition peak energy.

rate. For the second category, an effective way is to reduce the electron occupation of ground states at non- $E_{1}$ subbands.

\section{Conclusion}

In conclusion, we have demonstrated a GaAs/AlGaAs multipeak quantum cascade detector. The main response peak appeared at $8.9 \mu \mathrm{m}$ while four other response peaks from $4.5 \mu \mathrm{m}$ to $10.1 \mu \mathrm{m}$ were observed and analyzed. We confirmed that the multipeak performance comes from the complicated optical transition in the quantum cascade structure. This work is valuable for the design and optimization of QCD devices.

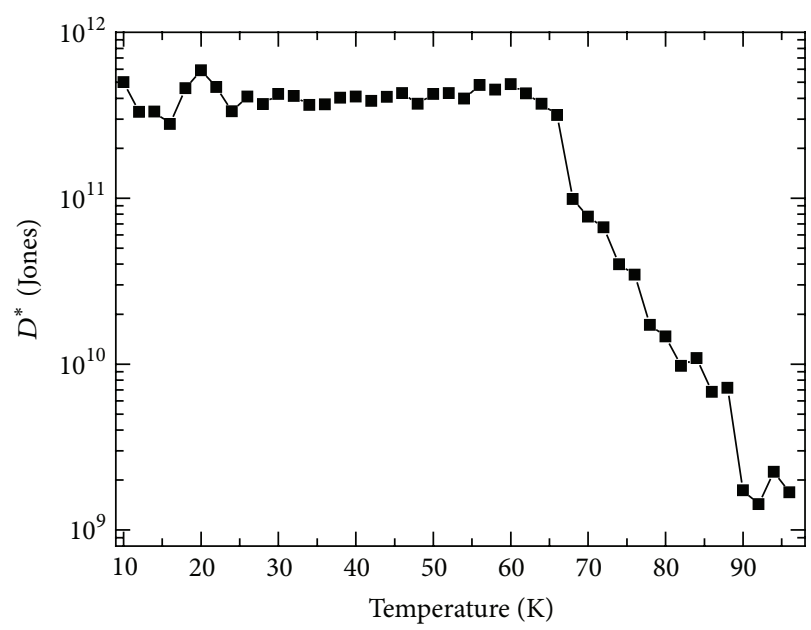

FIGURE 5: Peak wavelength detectivity.

\section{Conflict of Interests}

The authors declare that there is no conflict of interests regarding the publication of this paper.

\section{Acknowledgments}

This work was supported by the National Nature Science Foundation of China (Grant no. 61106092) and the State Key Development Program for Basic Research of China (Grants no. 2013CB632802).

\section{References}

[1] L. Gendron, C. Koeniguer, X. Marcadet et al., "Quantum cascade detection," in Electro-Optical and Infrared Systems: Technology and Applications, vol. 5612 of Proceedings of SPIE, 63, December 2004.

[2] Q. Li, Z. F. Li, N. Li et al., "High-polarization-discriminating infrared detection using a single quantum well sandwiched in plasmonic micro-cavity," Scientific Reports, vol. 4, article 6332, 2014.

[3] L. Gendron, C. Koeniguer, V. Berger, and X. Marcadet, "High resistance narrow band quantum cascade photodetectors," Applied Physics Letters, vol. 86, no. 12, Article ID 121116, 2005.

[4] A. Delga, L. Doyennette, V. Berger, M. Carras, V. Trinité, and A. Nedelcu, "Performances of quantum cascade detectors," Infrared Physics and Technology, vol. 59, pp. 100-107, 2013.

[5] D. Hofstetter, M. Graf, T. Aellen, J. Faist, L. Hvozdara, and S. Blaser, " $23 \mathrm{GHz}$ operation of a room temperature photovoltaic quantum cascade detector at $5.35 \mu \mathrm{m}$," Applied Physics Letters, vol. 89, no. 6, Article ID 061119, 2006.

[6] S.-Q. Zhai, J.-Q. Liu, X.-J. Wang et al., "19 $\mu \mathrm{m}$ quantum cascade infrared photodetectors," Applied Physics Letters, vol. 102, no. 19, Article ID 191120, 2013.

[7] S. Q. Zhai, J. Q. Liu, N. Kong et al., "Strain-compensated InPbased InGaAsInAlAs quantum cascade infrared detectors for 3-5 $\mu \mathrm{m}$ atmospheric window," in International Symposium on Photoelectronic Detection and Imaging 2011: Advances in Infrared Imaging and Applications, vol. 8193 of Proceedings of SPIE, Beijing, China, September 2011. 
[8] L. Gendron, C. Koeniguer, X. Marcadet, and V. Berger, "Quantum cascade detectors," Infrared Physics and Technology, vol. 47, no. 1-2, pp. 175-181, 2005.

[9] S. Sakr, P. Crozat, D. Gacemi et al., "GaN/AlGaN waveguide quantum cascade photodetectors at lambda approximate to $1.55 \mu \mathrm{m}$ with enhanced responsivity and similar to $40 \mathrm{GHz}$ frequency bandwidth," Applied Physics Letters, vol. 102, Article ID 011135, 2013.

[10] E. Bellotti and D. D’Orsogna, "Numerical analysis of HgCdTe simultaneous two-color photovoltaic infrared detectors," IEEE Journal of Quantum Electronics, vol. 42, no. 4, pp. 418-426, 2006.

[11] W.-D. Hu, X.-S. Chen, Z.-H. Ye, and W. Lu, "An improvement on short-wavelength photoresponse for a heterostructure $\mathrm{HgCdTe}$ two-color infrared detector," Semiconductor Science and Technology, vol. 25, no. 4, Article ID 045028, 2010.

[12] W. D. Hu, Z. H. Ye, L. Liao et al., "128 × 128 long-wavelength/ mid-wavelength two-color HgCdTe infrared focal plane array detector with ultralow spectral cross talk," Optics Letters, vol. 39, no. 17, pp. 5184-5187, 2014.

[13] J. Liu, N. Kong, L. Li et al., "High resistance AlGaAs/GaAs quantum cascade detectors grown by solid source molecular beam epitaxy operating above liquid nitrogen temperature," Semiconductor Science and Technology, vol. 25, no. 7, Article ID 075011, 2010

[14] B. F. Levine, "Quantum-well infrared photodetectors," Journal of Applied Physics, vol. 74, no. 8, p. R1, 1993.

[15] X. H. Liu, X. H. Zhou, N. Li et al., "Effects of bias and temperature on the intersubband absorption in very long wavelength GaAs/AlGaAs quantum well infrared photodetectors," Journal of Applied Physics, vol. 115, no. 12, Article ID 124503, 2014. 

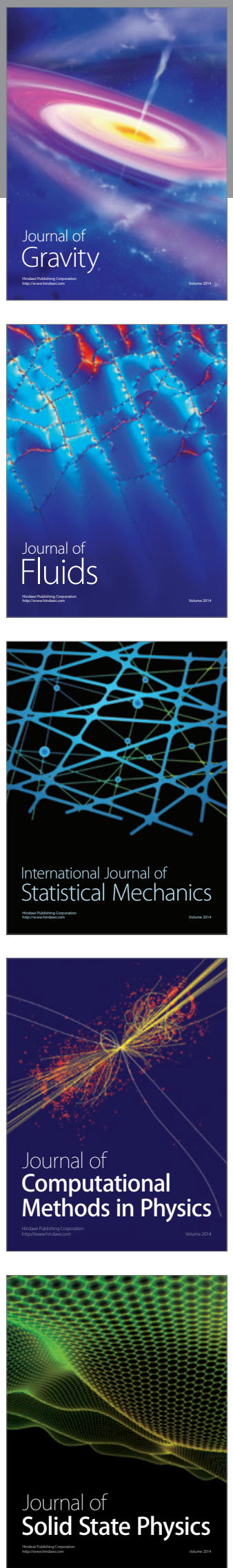

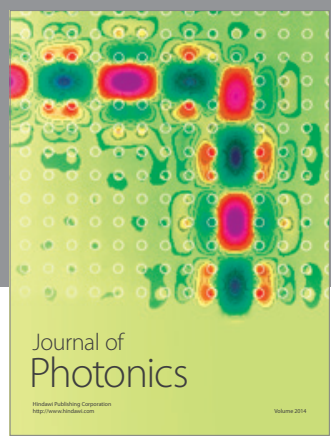

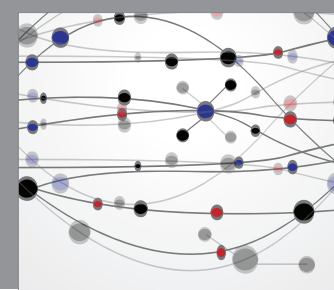

The Scientific World Journal

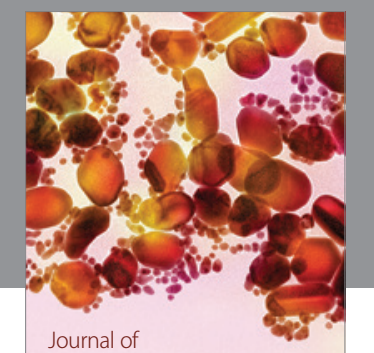

Soft Matter
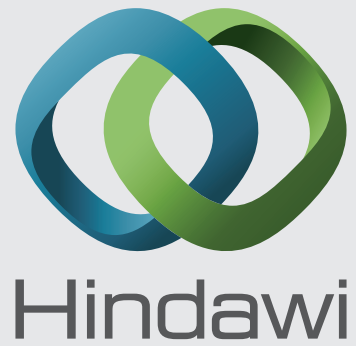

Submit your manuscripts at

http://www.hindawi.com
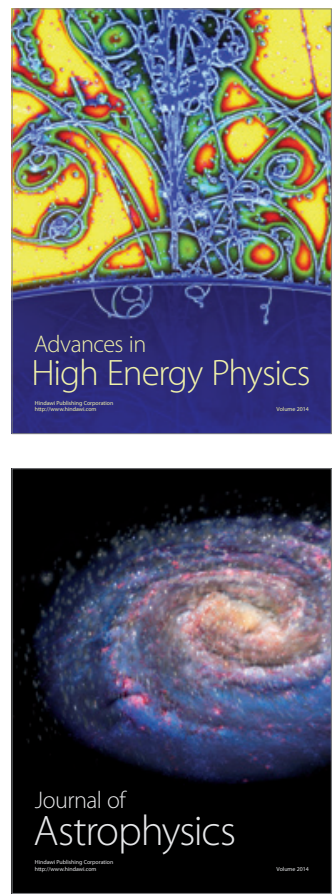
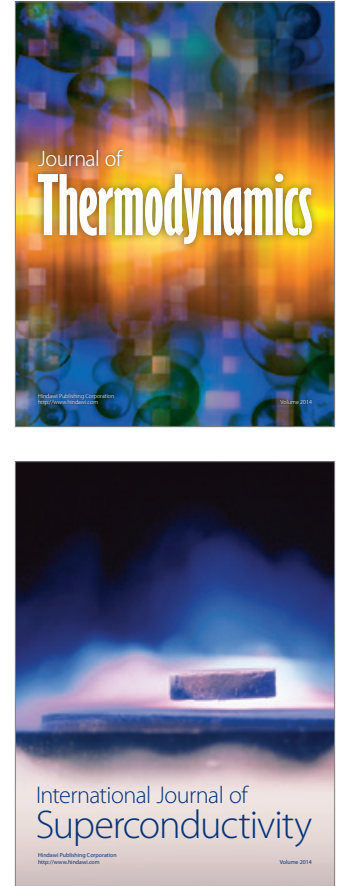
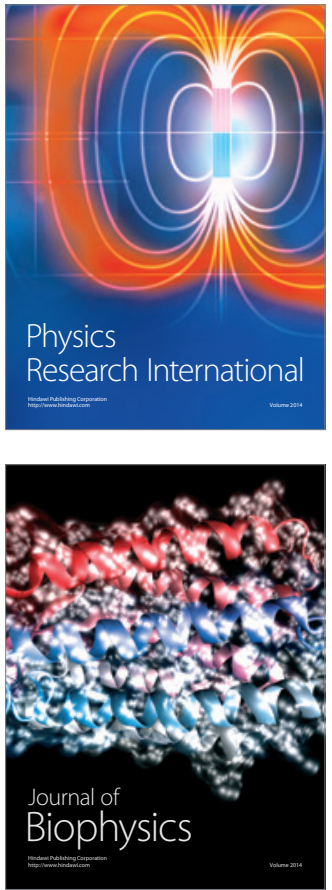
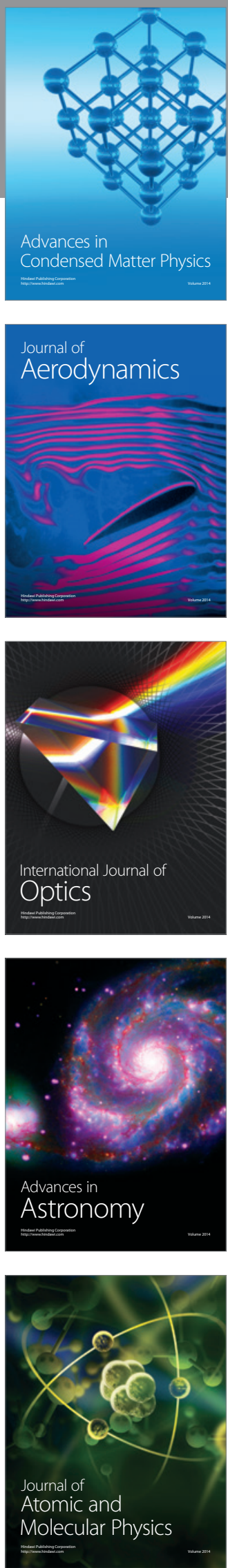